\title{
STUDY THE SPACE DEBRIS IMPACT IN THE EARLY STAGES OF THE NANO-SATELLITE DESIGN
}

\author{
Mohammed Chessab Mahdi \\ Al-Furat Al-Awsat Technical University -Iraq \\ $\underline{\text { mchessab@yahoo.com }}$
}

\begin{abstract}
The probability of KufaSat collisions with different sizes of orbital debris and with other satellites which operating in the same orbit during orbital lifetime was determined. Apogee/Perigee Altitude History was used to graph apogee and perigee altitudes over KufaSat lifetime. The required change in velocity for maneuvers necessary to reentry atmospheric within 25 years was calculated. The prediction of orbital lifetime of KufaSat using orbital parameters and engineering specifications as inputs to the Debris Assessment Software (DAS) was done, it has been verified that the orbital lifetime will not be more than 25 years after end of mission which is compatible with recommendation of Inter-Agency Space Debris Coordination Committee (IADC).
\end{abstract}

Keywords: Orbital Debris Mitigation, Mission Analysis, DAS, KufaSat, IADC, NanoSatellite.

\section{INTRODUCTION}

Orbital debris is anything made by man, orbiting the earth, and no serves a useful function such as parts of the space rocket, pieces of spaceships or equipment, old unusable satellites, and very small flecks of paint that will be liberated as a result of thermal stress or impacts of small particle. Space debris is generated in one of three ways: mission operations, accidental, and intentional (International Space University, Florida Institute of Technology, 2012). Space debris may be classified as belonging to one of four types of debris:

1. Fragmentation debris: It is space objects generated during breakups. Breakups are the events that separate an object into several smaller pieces with different initial velocities.

2. Mission-related debris: It is space objects may be released as a result of the functional operation of the satellite itself like explosive bolts, spring release mechanisms, in addition to eject protective coverings from payload and sensors. Also it includes resulting items from space flight like exhaust products from Solid Rocket Motors, and paint flakes.

3. Non-functional spacecraft: These are the functional spacecraft that ended their life after the mission is completed or terminated because of malfunction or failure and left in their origin orbits or transferred to another orbit farther or closer to the Earth.

4. Rocket bodies: Through spacecraft launching process, launch vehicle may leave four rocket bodies in different orbits that pass through it toward its final destination for high- 
altitude mission. The presence of these rocket bodies in orbits represents a major threat to functional spacecraft that used same orbits (National Academy of Sciences, 2011).

The risk of debris to the space operations depend on space operations nature and the region of the orbit that in which these operations occur. The damage caused by debris impact depends on the debris size and velocity of collision which vary with orbital altitude and inclination. It is very important to determine the risk to space operations from debris so; information about these orbital debris environments is needed .

In order to track the debris that can be considered large objects, a ground-based sensors network are used. There are two networks of ground based sensors in the world, first is the (SSN) which is United State Space Surveillance Network .It consists of group of optical sensors and radars (more than 20). Second is (SSS) which is the Russian Federation Space Surveillance It consist of 10 radar for tracking debris in lower orbits and 12 facilities (electrooptical and optical) for tracking debris high orbits.

The space debris monitor (SDM) is a large-area impact sensor for detect and measure micro- debris in the size of $100 \mu \mathrm{m}$ to a few millimetres in the near-Earth space environment using the concept of conductive strip lines. One or more of the stripes will be damaged and become non-conductive when a micro-debris particle, that have dimensions equal to or larger than dimensions of spatial separation of stripes, collides with the sensor film at a velocity enough to penetrate it. By monitoring the electrical resistant of the stripes it can be detect debris impacts, also it can be measure the size of debris particles that penetrated sensor film by detecting the number of damaged stripes (Committee on Space Debris, National Research Council, 1995). Figure 1 shows the types and number of debris that tracked by SSN (NASA JSC, 2014).

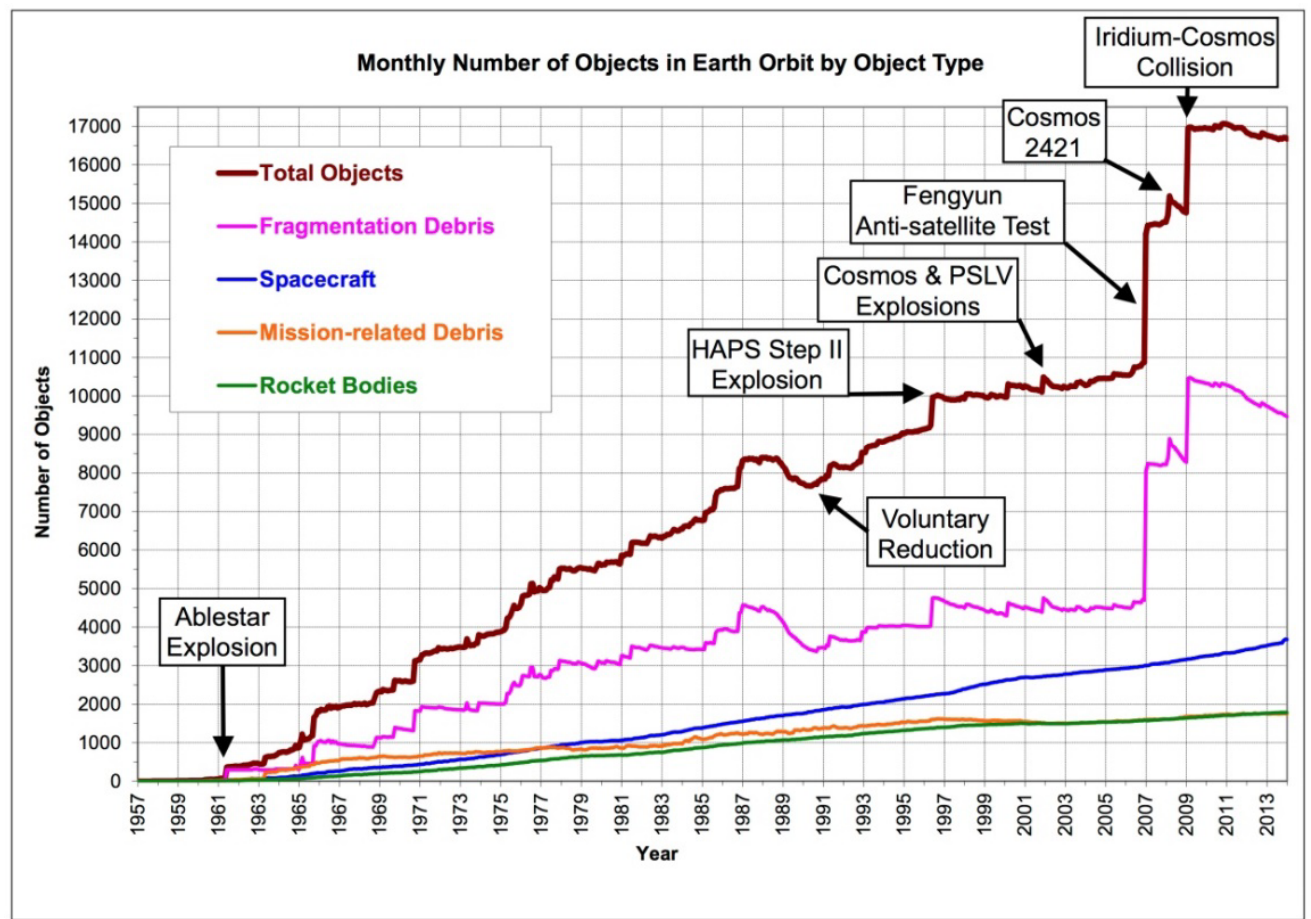

Fig. 1. Growth of orbital space object including space debris (NASA Orbital Debris program)

As a result of quickly growth in the number of satellites, the amount of orbital debris is growing rapidly. The estimated numbers of space debris orbiting Earth are (29 000) for large sizes greater than $10 \mathrm{~cm},(670000)$ for medium sizes greater than $1 \mathrm{~cm}$, and about $(170$ million) for small sizes greater than $1 \mathrm{~mm}$. Any of this space debris represents threat to 
operational spacecrafts in Earth orbit because this debris is travelling at very high orbital speeds, about $(7-8 \mathrm{~km} / \mathrm{s})$ in Low Earth Orbit (LEO).

Depending upon their perigee altitude satellites and other objects placed in LEO will remain in orbit for many years. Space debris in the higher altitude remains in orbit longer. Space debris at altitudes less than $600 \mathrm{~km}$ fall to the Earth after several years. Debris at an altitude of $800 \mathrm{~km}$ falls to the Earth after several decades. (NASA Orbital Debris program).

Over the past twenty years, LEO debris amount has increased rapidly, which led to increase the probability of collision that cause the generation of debris rate exceeds the rate at which debris deorbit, and fall into the atmosphere and burn up. This accumulation creates debris belts that make many orbits unusable. Figure 2 is an image that was generated by computer which illustrates the objects in LEO that are tracked by SSN, 95\% of these objects represent space debris.

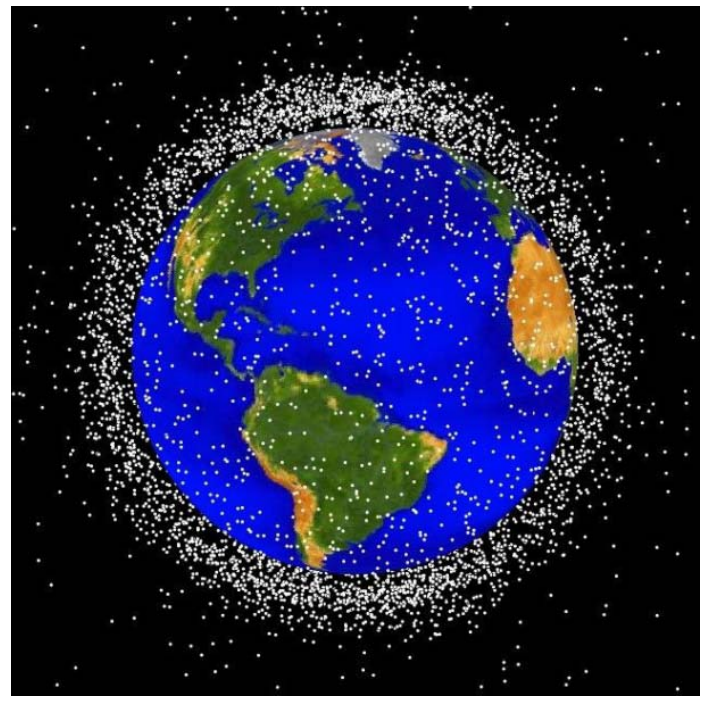

Fig. 2. Computer generated image of orbital debris in LEO. (NASA)

KufaSat is the first satellite of the University of Kufa, Iraq. It is developed within the framework of program called KufaSat Project, whose goal is to provide hands-on experience to aerospace students in cooperation with another three Iraqi universities (Al-Furat Al-Awsat Technical University, Al-Nahrain University, and Basrah University). The calculated Keplerian elements for KufaSat are listed in Table 1 (Mahdi M. Chessab, 2015).

Table 1. KufaSat orbital elements

\begin{tabular}{|c|c|c|}
\hline Field & Value & Unit \\
\hline Semi-Major Axis (SMA) & 6978 & $\mathrm{~km}$ \\
\hline Eccentricity (ECC) & 0 & ---- \\
\hline Inclination (INC) & 97 & $\mathrm{deg}$ \\
\hline Argument of Perigee (AOP) & 150 & $\mathrm{deg}$ \\
\hline Right Ascension of Ascending Node (RAAN) & 0 & $\mathrm{deg}$ \\
\hline True Anomaly (TA) & 10 & $\mathrm{deg}$ \\
\hline
\end{tabular}

Physical parameters of KufaSat and its orbit are listed in Table 2 (Mahdi, M. C. 2014). 
Table 2. KufaSat Physical parameters

\begin{tabular}{|c|c|}
\hline Spacecraft shape & Cube \\
\hline Spacecraft dimensions & $(10 \times 10 \times 10) \mathrm{cm}$ \\
\hline Spacecraft mass & $1.3 \mathrm{~kg}$ \\
\hline Orbit type & Polar LEO \\
\hline Altitude & $600 \mathrm{~km}$ \\
\hline Orbital period & 96.684 minutes \\
\hline Mission lifetime & 3 years \\
\hline
\end{tabular}

\section{ORBITAL DEBRIS MITIGATION GUIDELINES}

Several orbital debris mitigation guidelines have been issued by various organizations NASA, U.S Government, the Federal Communications Commission (FCC), and the InterAgency Space Debris Coordination Committee (IADC). IADC Space Debris Mitigation Guidelines will be summarized in this work.

The (IADC) is an international forum whose aim is to coordinate activities and efforts to deal with the all types of debris (natural and man-made) in orbit around the Earth.

In order to eliminate or minimize creation of debris during operations, (IADC) recommends debris mitigation guidelines and focus attention to cost effectiveness during all the phases of design the launch vehicles and spacecraft.

The IADC Guidelines describe some current practices which have been evaluated and identified for limiting the creation of space debris in the environment. Limitation of space debris which be liberated during operations, minimization of the probability of break-ups inorbit, disposal end mission, and prevent collisions that occur on-orbit represent the overall environmental impact of the missions in these guidelines.

\subsection{IADC MITIGATION MEASURES}

The main Mitigation measures in IADC guidelines are:

1-Limit Debris Released during Normal Operations: Reduction the generation of debris during normal operations is requirement for spacecraft manufacturers, so all spacecraft must be designed in a way that not allow releasing debris during normal operations. If that is not possible, the space debris must be limited.

2-Minimize the probability of break-ups in-orbit: The main factors which cause On-orbit breaks-ups can be prevented using the following measures:

- Minimize the probability of (after mission) break-ups as a result of stored energy

- Minimize the probability for break-ups during different phases operation

- Avoidance the harmful activities and deliberate destruction

3-Post Mission Disposal: A spacecraft should be left in an orbit where drag and other perturbations will limit lifetime after completion of operations. To avoid collisions between spacecraft and orbital debris the (IADC) recommended that the appropriate lifetime limit is 25-year.

4-Prevention of On-Orbit Collisions: Collision with objects could cause loss of control for this reason the spacecraft design should estimate and limit probability of collision with objects (Inter-Agency Space Debris Coordination Committee, 2007). 


\section{DEBRIS ASSESSMENT SOFTWARE (DAS)}

The Debris Assessment Software (DAS) version 2.0.2 is used in this work. DAS is used by NASA in performing space debris estimates. DAS 2.0.2 uses the NASA propagator "PROP3D" to keep accuracy of integration over long periods of propagation with acceptable computation speed. Solar and Lunar gravity, Solar Radiation Pressure (SRP), Atmospheric drag, and Earth's gravity field with Zonal harmonics (J2, J3, and J4) are the perturbations included in the DAS orbit propagator. The atmospheric model used in DAS was the Jacchia 1976 Standard Model. The drag coefficient is supposed to be 2.2 and the reflection coefficient value for (SRP perturbation) is supposed to be 1.25. The major functions of DAS are divided into three sections: mission editor, requirement assessments, and associated science and engineering utilities. The mission information must be entered into the mission editor.

Requirement assessments section of DAS includes routines to estimate the acquiescence to the mission with each NASA requirement about debris limiting. Science and engineering utilities provide a number of functions useful for mission planning and allow analyzing some aspects of orbit/mission design (NASA, 2012).

\section{SIMULATION AND RESULTS}

Science and engineering utilities include six categories: on-orbit collision, analysis of postmission disposal maneuver, orbit evolution analysis, delta- $\mathrm{V}$ postmission maneuver analysis, orbit to orbit transfer, and other utilities (Inter-Agency Space Debris Coordination Committee, 2007). The first four categories of science and engineering utilities will be applied to study the space debris impact on KufaSat Nano-Satellite.

\section{a) On-Orbit Collisions}

Probability of debris impacts versus a verity of factors can be graphed to provide a visual aid. Figure 3 explains the relation between Debris Impacts against Orbit Altitude, Figure 4 explains the relation between Debris Impacts against Debris Diameter, and Figure 5 explains the relation between Debris Impacts against Date. These utilities assist in the estimation of acquiescence with Requirement 5 (limitation the operating systems to be debris sources as a result of collisions).

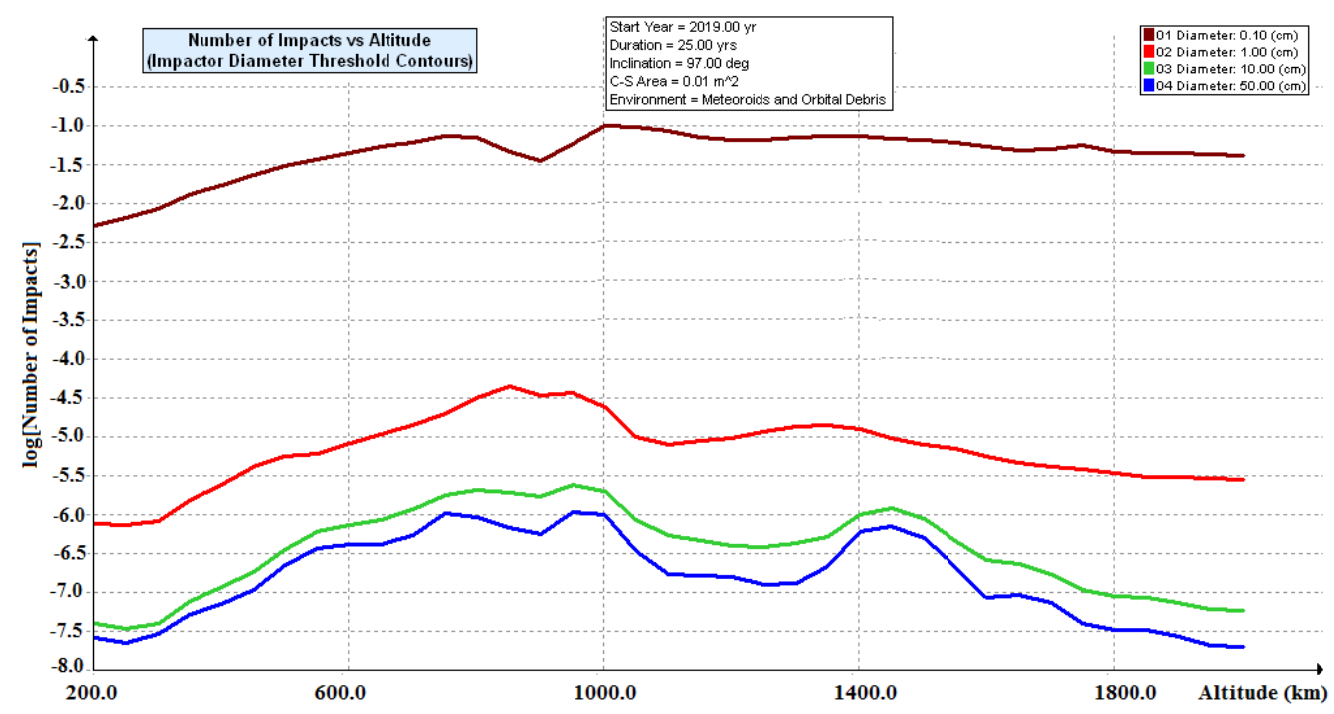

Fig. 3. Debris Impacts against Orbit Altitude 


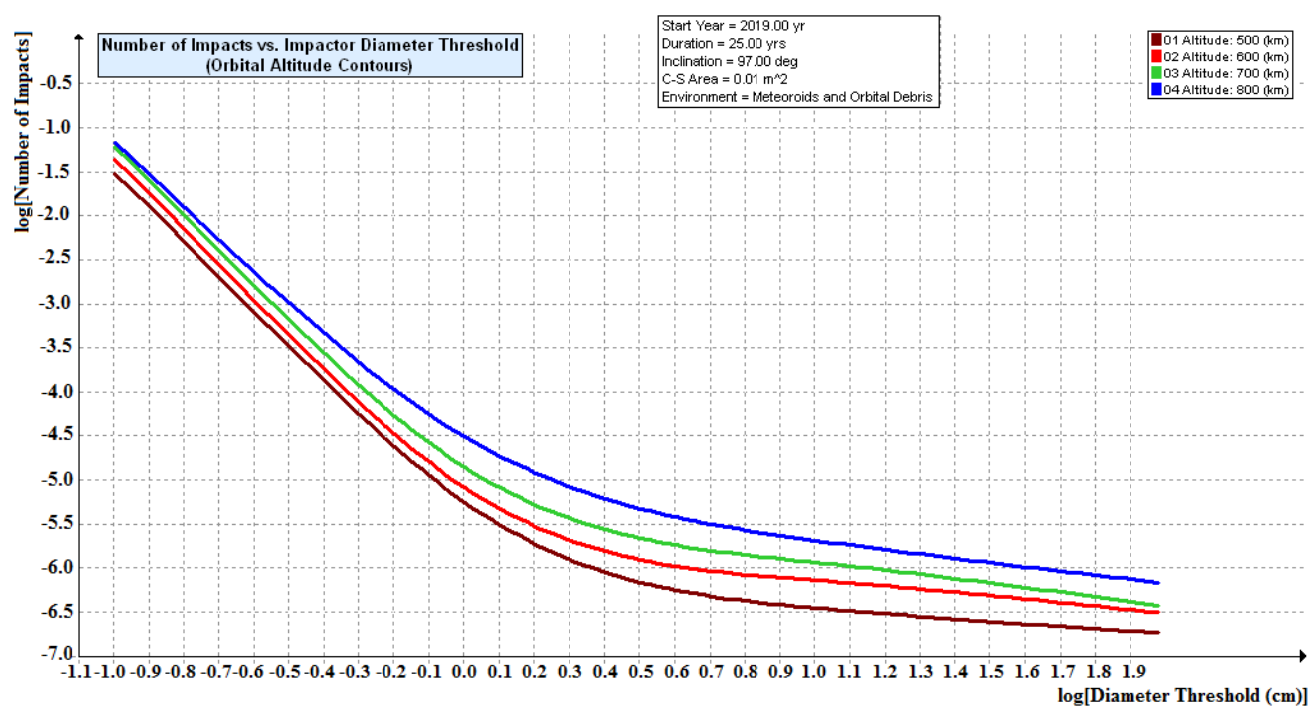

Fig. 4. Debris Impacts against Debris Diameter

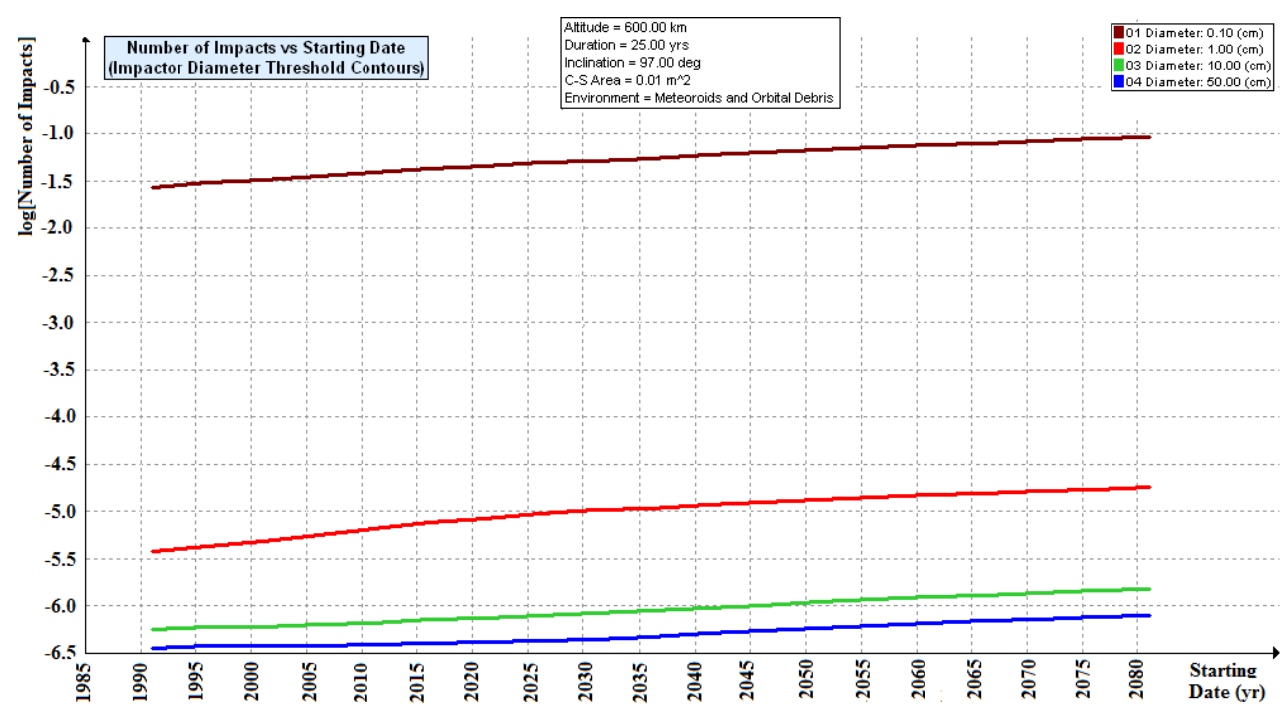

Fig. 5. Debris Impacts against Date

b) Analysis of Postmission Disposal Maneuvers

These utilities address the required orbital maneuver to transfer from low earth orbit to a decay orbit for disposal by entry atmospheric. This may assist in determining the cost of deorbit maneuvers. Figure 6 explains disposal by atmospheric reentry. These utilities assist in the estimation acquiescence with Requirement 6 (end mission disposal). 


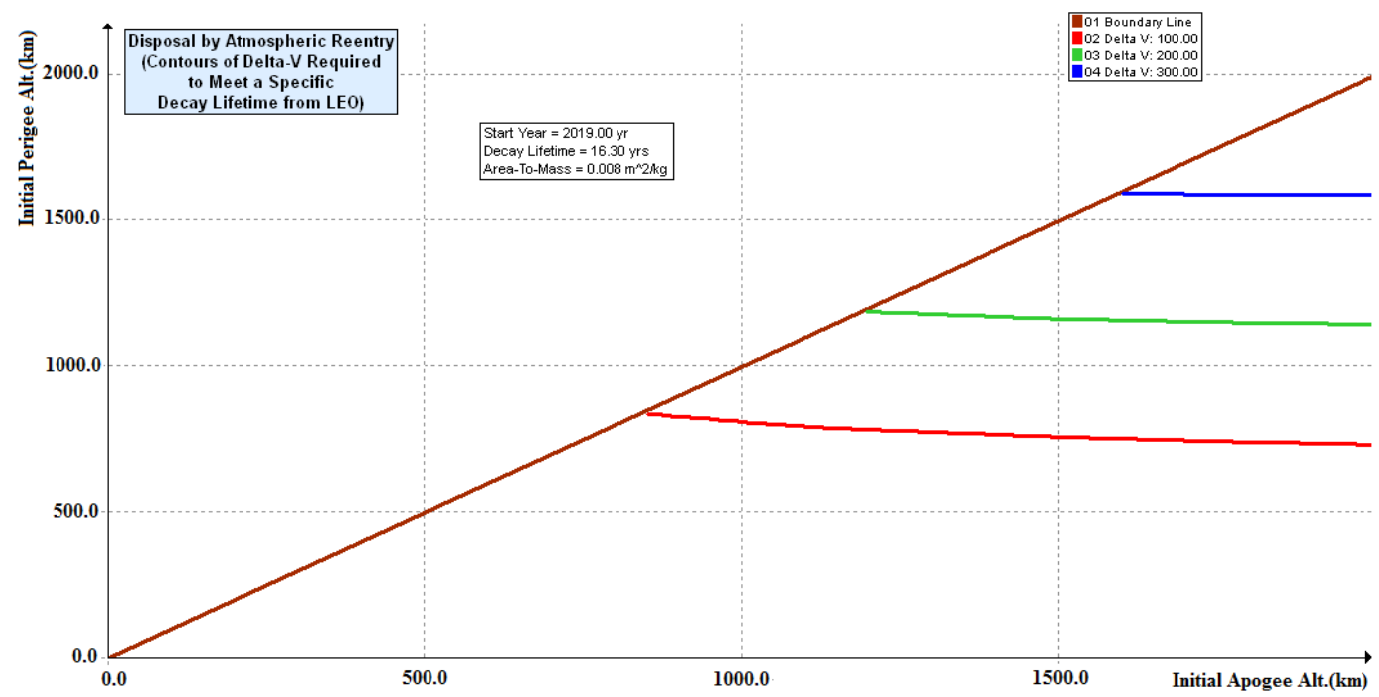

Fig. 6. Disposal by Atmospheric Reentry

c) Orbit Evolution Analysis

This group consist of two utilities, first is Altitudes History which is used to graph apogee and perigee altitudes over object lifetime as shown in Figure 7. Second, Orbit Lifetime and Dwell Time that can calculate the Lifetime and low earth orbit Dwell Time of spacecraft in a given orbit. These utilities assist in the estimation acquiescence with Requirement 6 (end mission disposal).

For KufaSat it is found that the calculated Orbital Lifetime from year 2019 as a start year equal to 16.356 years. The year in which KufaSat either came back and entered the atmospheric or exceeded the limit of propagation time is 2035. Table 3 shows the effect of varying the area to mass ratio on orbital lifetime of KufaSat with assuming 2019 is the start year.

Table 3. Area to mass ratio vs. Orbital lifetime

\begin{tabular}{|c|c|c|}
\hline $\begin{array}{c}\text { Area to } \\
\text { mass ratio }\end{array}$ & $\begin{array}{c}\text { Orbital } \\
\text { lifetime }\end{array}$ & $\begin{array}{c}\text { Last year of } \\
\text { propagation }\end{array}$ \\
\hline 0.005 & 26.683 & 2045 \\
\hline 0.006 & 24.241 & 2043 \\
\hline $\mathbf{0 . 0 0 7 6 9 2 3}$ & $\mathbf{1 6 . 3 5 0}$ & $\mathbf{2 0 3 5}$ \\
\hline 0.009 & 15.086 & 2034 \\
\hline 0.010 & 14.286 & 2033 \\
\hline 0.015 & 6.779 & 2025 \\
\hline 0.020 & 5.235 & 2024 \\
\hline 0.030 & 4.233 & 2023 \\
\hline 0.100 & 2.689 & 2021 \\
\hline 0.500 & 1.884 & 2020 \\
\hline
\end{tabular}




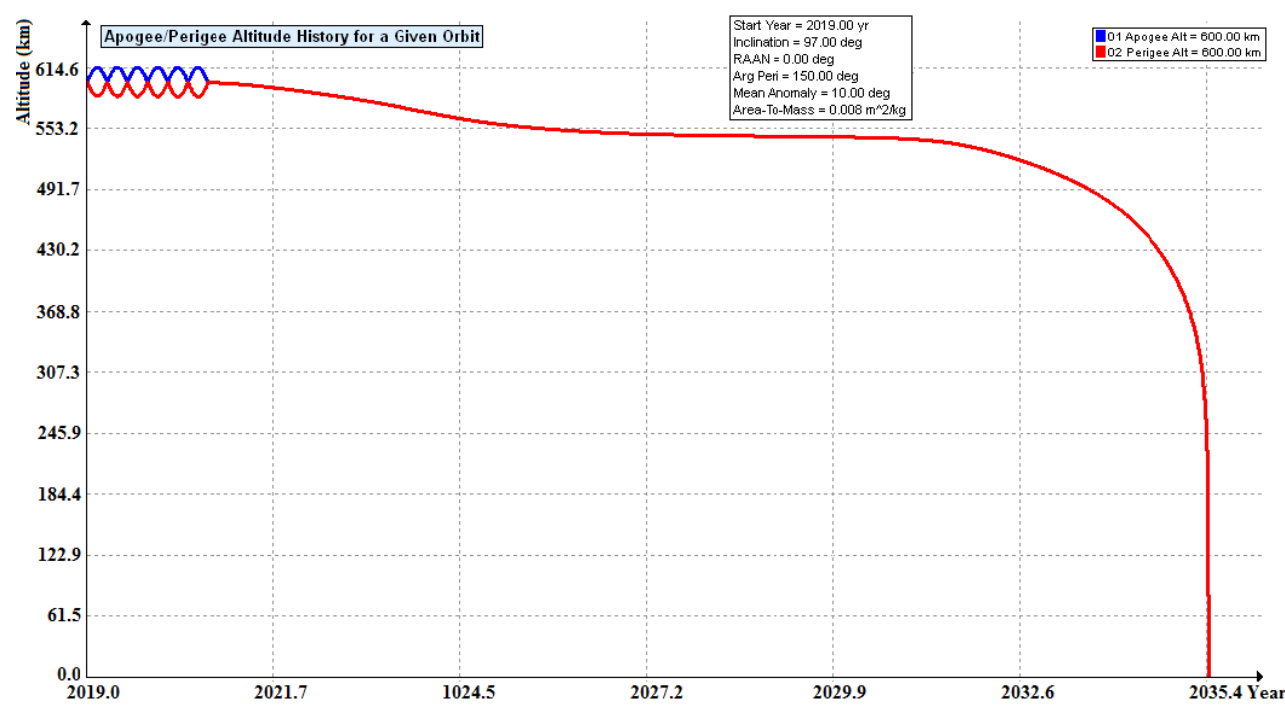

Fig. 7. Altitude against Year for a Specific Orbit

d) Delta-V for Postmission Maneuver

By these utilities, the velocity change needed for maneuvers necessary to achieve coming back to atmospheric within limit of lifetime ( 25 years), can be calculated. This group consists of two utilities:

1- Delta-V for Decay Orbit Given Orbital Lifetime: plots (area/mass) ratio corresponding to the change in velocity required to transfer an object, with a given orbital lifetime, from low earth orbit to a decay orbit. Cost of deorbiting a vehicle can be exploring from the plot. Figure 8 represents the output of this utility for KufaSat.

2- Delta-V for Decay Orbit Given Area-To-Mass: plots lifetime corresponding to the change in velocity required to transfer an object, with a given (area/mass) ratio, from low earth orbit to a decay orbit. Cost of deorbiting a vehicle over a range of decay lifetimes can be exploring from the plot. Figure 9 represents the output of this utility for KufaSat.

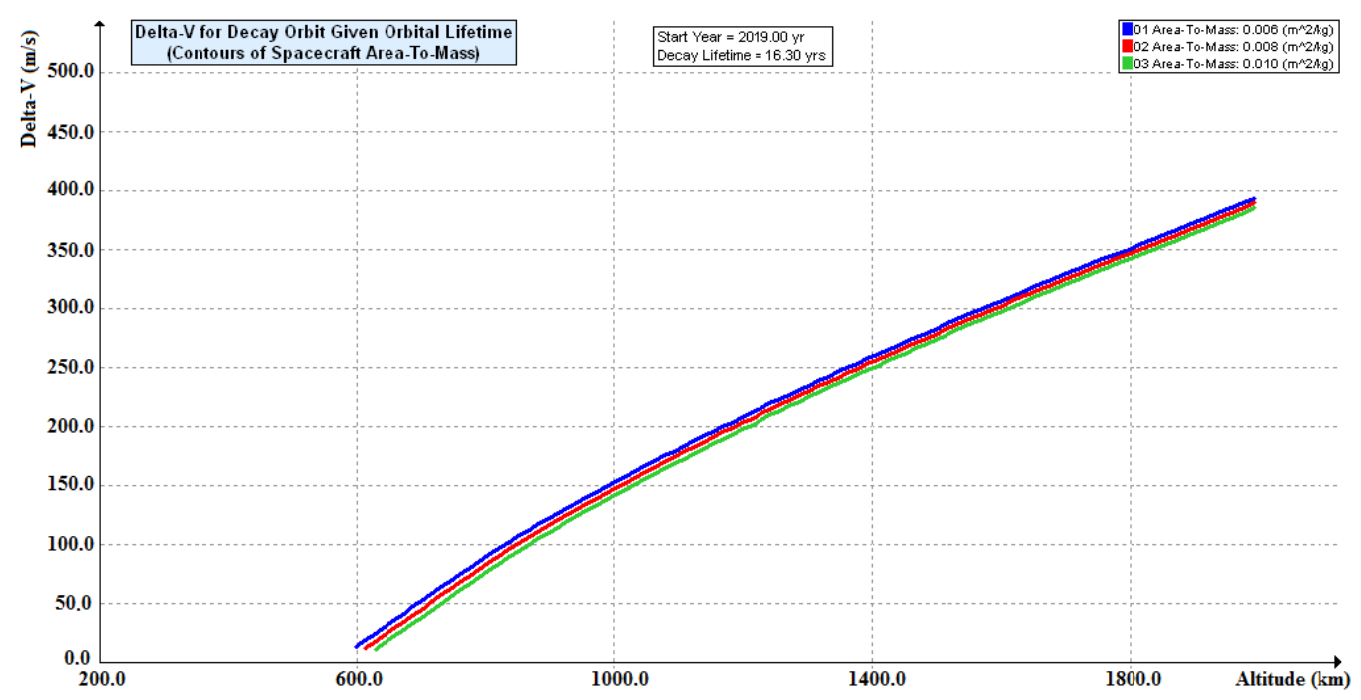

Fig. 8. Delta-V against Altitude Specific Orbital Lifetime 


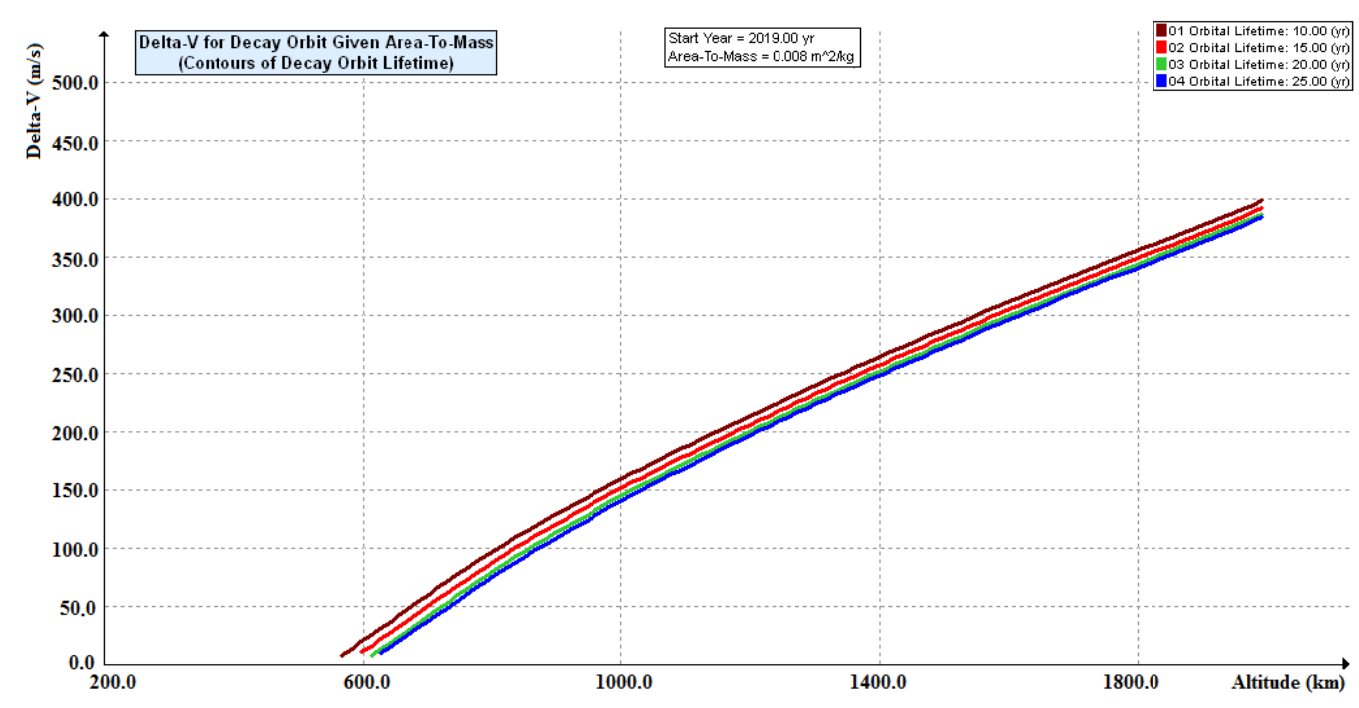

Fig. 9. Delta-V against Altitude Specific (Area/Mass)

\section{CONCLUSIONS}

Space debris mitigation is required for each space mission to ensure of compliance with requirements and standards in addition to ensure that the spacecraft can resist the space debris environment during the mission lifetime. One of important aspect is estimation and limitation the chances of collision with different sizes of orbital debris and with other satellites which operating in the same orbit during orbital lifetime which cause uncontrollable state to prevent end mission disposal. KufaSat mission analysis was conducted using the Debris Assessment Software (DAS). By this software the probability of collisions with different sizes of known objects during orbital lifetime of KufaSat was determined.

By using orbital parameters and engineering specifications of KufaSat as inputs to DAS it has been verified that the orbital lifetime will not be more than allowable limit ( 25 years) after end of mission.

\section{REFERENCES}

Committee on Space Debris, National Research Council (1995), ORBITAL DEBRIS: A Technical Assessment, National Academies Press.

International Space University, Florida Institute of Technology. (2012), Operations and Service Infrastructure for Space Melbourne, Florida, USA, SSP.

Inter-Agency Space Debris Coordination Committee (IADC) Website, Available on http://www.iadc-online.org/index.cgi?item=home

Inter-Agency Space Debris Coordination Committee, (2007) IADC Space Debris Mitigation Guidelines, Revision 1, IADC-02-01.

Mahdi M. Chessab (2015) Orbit Design and Simulation for KufaSat Nano-Satellite, Artificial Satellites Journal, Vol. 50, No. 4.

Mahdi, M. C. (2014) Design and Implementation of an Effective Electrical Power System for Nano-Satellite, International Journal of Scientific \& Engineering Research, Volume 5, Issue 5.

NASA JSC. (January 2014) Orbital Debris Quarterly News, Volume 18, Issue 1. 
NASA Orbital Debris program Office Orbital Debris Frequently Asked Questions, Available on http://orbitaldebris.jsc.nasa.gov/faq.html\#3

NASA (2012), Debris Assessment Software User's Guide Version 2.0 Lyndon B. Johnson Space Center, Houston, Texas 77058.

National Academy of Sciences. (2011) Limiting Future Collision Risk to Spacecraft: An Assessment of NASA's Meteoroid and Orbital Debris Programs, National Academies Press, Washington, D.C.

Received: 2016-05-09,

Reviewed: 2016-07-01, by Mohammed J. Al-Bermani, 2016-11-03, by Adnan F. Hassan, and 2016-11-30,

Accepted: 2016-12-06. 